\title{
Comprehensive Genetic Variant Discovery in the Surfactant Protein B Gene
}

\author{
AARON HAMVAS, DANIEL J. WEGNER, CHRISTOPHER S. CARLSON, KELLY R. BERGMANN, MICHELLE A. TRUSGNICH, \\ LUCINDA FULTON, YUMI KASAI, PING AN, ELAINE R. MARDIS, RICHARD K. WILSON, AND F. SESSIONS COLE
}

\begin{abstract}
Edward Mallinckrodt Department of Pediatrics [A.H., D.J.W., M.A.T., F.S.C.], Genome Sequencing Center [L.F., Y.K., E.R.M., R.K.W.], Department of Genetics [K.R.B., P.A.], Washington University School of Medicine and St. Louis Children's Hospital, St. Louis, Missouri 63110; Division of Public Health Sciences [C.S.C.], Fred Hutchinson Cancer Research Center, Seattle, Washington 98109
\end{abstract}

\begin{abstract}
Completely penetrant mutations in the surfactant protein B gene $(S F T P B)$ and $>75 \%$ reduction of $S F T P B$ expression disrupt pulmonary surfactant function and cause neonatal respiratory distress syndrome. To inform studies of genetic regulation of SFTPB expression, we created a catalogue of $S F T P B$ variants by comprehensive resequencing from an unselected, population-based cohort $(n=1,116)$. We found an excess of low-frequency variation [81 SNPs and five small insertion/deletions (in/dels)]. Despite its small genomic size $(9.7 \mathrm{~kb})$, SFTPB was characterized by weak linkage disequilibrium (LD) and high haplotype diversity. Using the HapMap Yoruban and European populations, we identified a recombination hot spot that spans $S F T P B$, was not detectable in our focused resequencing data, and accounts for weak LD. Using homologybased software tools, we discovered no definitively damaging exonic variants. We conclude that excess low-frequency variation, intragenic recombination and lack of common disruptive exonic variants favor complete resequencing as the optimal approach for genetic association studies to identify regulatory $S F T P B$ variants that cause neonatal respiratory distress syndrome in genetically diverse populations. (Pediatr Res 62: 170-175, 2007)
\end{abstract}

$\mathrm{T}$ he 9.7-kb SFTPB (GeneID: 6439 Locus tag: HGNC 10801; MIM 178640) encodes a 79-amino-acid hydrophobic protein that is critical for function of the pulmonary surfactant (1). Functional pulmonary surfactant, a phospholipidprotein mixture that lines alveoli at the air-liquid interface, maintains alveolar patency at end expiration and is required for successful fetal-neonatal pulmonary transition. Studies in human newborn infants with rare recessive loss of function SFTPB mutations have demonstrated that genetic disruption of SFTPB expression is completely penetrant and lethal due to dysfunction of the pulmonary surfactant (2,3). Studies in conditionally regulated murine lineages and human infants indicate that $>75 \%$ reduction in SFTPB expression is sufficient to cause surfactant dysfunction and respiratory distress $(4,5)$. To provide a catalogue of $S F T P B$ variants [single nucleotide polymorphisms (SNPs) or in/dels] for use in sta-

Received December 6, 2006; accepted March 15, 2007.

Correspondence: F. Sessions Cole, M.D., St. Louis Children's Hospital, One Children's Place, St. Louis, MO 63110; e-mail: cole@kids.wustl.edu.

This work was supported by grants from the National Heart, Lung, and Blood Institute (RO1 HL 065174 to F.S.C., RO1 HL 065385 to A.H.), from the National Human Genome Research Institute (U54 HG 003079 to R.K.W.), from the Children's Discovery Institute of St. Louis Children's Hospital (F.S.C. and A.H.), and from the Saigh Foundation (F.S.C. and A.H.). tistical and functional studies of $S F T P B$ regulation, we used high-throughput, comprehensive resequencing of SFTPB in a cohort of sufficient size $(n=1116)$ to detect low frequency variants. We report an excess of low-frequency variation, high rates of intragenic recombination, and a lack of common damaging exonic variants. Our results suggest that comprehensive resequencing will likely be advantageous over tagSNP genotyping approaches in genetic association analysis of SFTPB.

\section{METHODS}

Automated amplification and sequencing. We extracted genomic DNA from 1116 Guthrie cards collected for newborn screening by the Missouri Department of Health and Senior Services (DHSS) (6). We linked each DNA sample anonymously to clinical characteristics in a vital statistics (birth-death certificate) database maintained by the Missouri DHSS to determine ethnicity. Using small amplicons $[<500$ base pairs (bp)], robotic high-throughput automated processes, and BigDye terminator sequencing chemistry (7), we bidirectionally sequenced $S F T P B$, including $1.8 \mathrm{kB}$ of the promoter region, $1.1 \mathrm{kB}$ of exonic sequence (all 10 translated exons), and $5.9 \mathrm{kB}$ that includes all intervening intronic sequence except 380 bp (genomic position 16492028 ) in intron 4 . We omitted part of intron 4 due to the inability of BigDye terminator sequencing chemistry to resolve variable numbers of dinucleotide repeats in this region (8). We also omitted one untranslated exon (exon 11), and its preceding intron (intron 10). All amplification and sequencing primers and conditions are available at http://genome.wustl.edu/activity/med_seq/ primers.cgi. We used software applications (Phred, Phrap, PolyPhred, and Consed) to call bases, assemble contigs, and scan sequencing chromatograms for variation (http://www.phrap.org/phredphrapconsed.html).

To assess overall sequence quality, we used a quality-averaging program (J. Sloan, University of Washington) to quantify Phred score at each base across SFTPB (Fig. 1). Because of variation in trace file quality, analysts reviewed and confirmed or edited all polymorphic sites identified by Polyphred, sites with in/dels, and all sites previously identified as polymorphic in dbSNP in each individual. After manual polymorphism validation, we extracted genotypes for each DNA sample at the confirmed polymorphic sites for analysis. An average of $90 \%$ of genotypes were called in each individual using a minimum Phred score of 20.

False-positive and -negative rates of SNP discovery. Because of the high proportion of sequence variation attributable to rare polymorphic sites, we were concerned that SNP detection errors might bias our analysis. Systematic comparison of the results from two independent analysts identified $0.99 \%$ of calls as discrepant (452/45,505 genotypes): $67 \%$ of these were judged as false-positive calls $(301 / 452)$ in low-quality (Phred score $<20$ ) data, and all discrepant calls were classified as missing data. Using an independent genotyping method, Taqman (9), we compared genotypes at five high-frequency polymorphic sites in 558 individuals to the genotypes called from sequence data and found 27 discrepant calls in 2790 genotypes, with 10 confirmed

Abbreviations: in/del, insertion/deletion; LD, linkage disequilibrium; MAF, minor allele frequency; SFTPB, surfactant protein B gene; SNP, single nucleotide polymorphism 


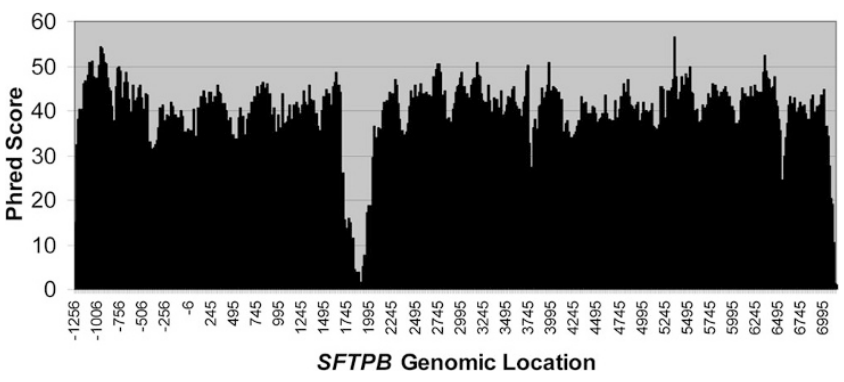

Figure 1. Average Phred score by genomic location in SFTPB. Average Phred score calculated and averaged at each site on finished $S F T P B$ sequence. Sequence quality in intron 4 was low due to the inability of BigDye sequencing chemistry to resolve multiple CA repeats.

Taqman heterozygotes, for a false-negative heterozygote detection rate of $0.36 \%$. Next, we reamplified and resequenced all heterozygous sites identified in fewer than three individuals ( 41 genotypes in 49 individuals) with different primer sets and confirmed genotypes at all these sites. Finally, we examined base calls and sequence quality (Phred score) at 42 sites polymorphic in other cohorts but not in this cohort (45,780 genotypes). Of the 41,555 genotypes with high-quality (Phred score $>20$ ) sequence, we found no rare alleles missed by chromatogram analysis $(0 \%)$. We could not call the remaining 5317 genotypes $(11.6 \%)$ due to low-quality chromatograms in those specific samples. These results suggest false-positive and -negative rates of less than $1 \%$.

LD, haplotype estimation, recombination rate, and hot spot location determination. LD is a measure of the allelic correlation between two SNPs. Several LD statistics are available (10); $\mathrm{D}^{\prime}$ is the ratio of the observed LD to the strongest possible LD given the allele frequencies of the SNPs. $\left|\mathrm{D}^{\prime}\right|=1$ when there is no detectable recombination between SNPs. Haplotypes are patterns of alleles across multiple SNPs along a single chromosome. We used PHASE (v. 2.1) to infer haplotypes computationally from genotypes within each racial group $(11,12)$. To assess whether haplotypes of common variants [minor allele frequency (MAF) $>5 \%$ ] can predict genotype at low-frequency $S F T P B$ alleles, we used HAPLOVIEW (v. 3.31) (http://www.broad.mit.edu/ $\mathrm{mpg} /$ haploview/) in aggressive mode to select a minimal set of tagSNPs such that all other SNPs were strongly correlated $\left(r^{2} \geq 0.8\right)$ with either a tagSNP or a haplotype of several tagSNPs (13). We used PHASE to estimate background recombination rate, determine hot spot location, and compute Bayes factors (BFs) as previously described (14) for either intragenic SNPs with MAF $>5 \%$ or for HapMap SNPs (MAF $>5 \%$ ) within $50 \mathrm{kB}$ of $S F T P B$ (data release \#21 as of July 2006) (http://www.hapmap.org). BFs are likelihood ratios of the probability of the observed data assuming a recombination hotspot divided by the probability of the data assuming uniform recombination across the region. A BF of 10 suggests that the haplotype data at a genomic location are 10 times more likely to be consistent with the presence of hot spot than the absence of a hot spot, and a BF of $>10$ is substantive evidence for the presence of a recombination hot spot.

Molecular evolution. Discovery of genomic regions under selective pressure may help inform genetic association studies because evolutionarily constrained sequences are presumably functional. We used three statistical strategies to screen $S F T P B$ for selective pressure. To assess whether genetic variation in regions of $S F T P B$ was consistent with neutral evolution, we used two statistical tests of observed sequence diversity against theoretical predictions for neutral sequence, Tajima's D (15) and the Fu and Li D* (16).
Tajima's D compares two descriptive statistics $(\theta$ and $\pi)$ for sequence diversity: $\theta$ is based on the number of chromosomes screened and the number of polymorphisms observed in SFTPB (17), whereas $\pi$ is based on the number of chromosomes screened and the average allele frequency of the polymorphisms identified $(18,19)$. We used SLIDER (http://genapps.uchicago.edu/ slider/index.html) to calculate Tajima's D. The Fu and Li D* compares $\pi$ with a third sequence diversity statistic derived from the number of singleton polymorphisms observed (SNPs with the rare allele observed only once in the data) (19).

We also characterized selection pressure by using the ratio of nonsynonymous to synonymous substitution rates (dN/dS) calculated from the observed SNPs using SNAP (Synonymous/Nonsynonymous Analysis Program) (http:// www.hiv.lanl.gov/content/hiv-db/SNAP/WEBSNAP/SNAP.html) (20,21). A $\mathrm{dN} / \mathrm{dS}$ ratio $>1$ suggests more nonsynonymous substitutions than expected under the neutral model and is evidence of positive selection, whereas a $\mathrm{dN} / \mathrm{dS}$ ratio $<1$ is evidence of purifying selection against some amino acid replacement mutations.

The third statistic that we used was the MacDonald-Kreitman test (22), which compares the within-species $\mathrm{dN} / \mathrm{dS}$ ratio for polymorphism in our sample against the between-species ratio for fixed differences (23) (http:// www.ebi.ac.uk/clustalw/).

Statistical methods and Human Studies Committee approval. We analyzed all data using Statistical Analysis System (v. 9.3.1) (SAS, Inc., Cary, NC). The Human Research Protection Office at the Washington University Medical Center and the Institutional Review Board at the Missouri DHSS reviewed and approved this study.

\section{RESULTS}

Genetic variant discovery. We were unable to screen 380 bp of intron 4 due to a highly polymorphic CA repeat region. In the remaining sequence, we found 86 polymorphic sites including 81 SNPs and five small in/dels (9.8 polymorphic sites per 1000 bp of $S F T P B$ reference sequence), with similar frequencies in the promoter (eight per $1000 \mathrm{bp})$, introns (10 per $1000 \mathrm{BP}$ ), and exons (12 per $1000 \mathrm{BP})\left(\chi^{2}\right.$ analysis, $p=$ $0.7)$ (Table 1). The overall SNP density was $9.2 / 1000 \mathrm{bp}$. The Phred scores within 10 bp of each polymorphic site (37 \pm 6$)$ (mean $\pm \mathrm{SD}$ ) were excellent, suggesting that sequence quality did not limit genetic variant discovery (Fig. 1). The average number of polymorphic sites per individual was greater in African-Americans than other races (all $p<0.01$ ) (Table 1). The race-specific relative genotype frequencies at each polymorphic site did not differ significantly from Hardy-Weinberg prediction (all $p>0.05$ ). The majority of variant sites in SFTPB are low frequency: 67 of 86 sites had MAF $<5 \%$. Potentially disruptive variants were also rare: eight of nine nonsynonomous variants and six of seven intronic SNPs within $20 \mathrm{bp}$ of an intron-exon junction were rare. To determine whether nonsynonymous SNPs might disrupt surfactant

Table 1. SFTPB polymorphic sites by race in the Missouri cohort

\begin{tabular}{lccccrrr}
\hline \multicolumn{1}{c}{ Race } & Private variants & Promoter & Intron & Intron/exon junction & Exon (S/NS) & Total & SNPs/individual \\
\hline African-American $(n=197)$ & 18 & 12 & 38 & 7 & $(4 / 6)$ & 60 & $6.4 \pm 2.9$ \\
European-American $(n=875)$ & 24 & 11 & 44 & 5 & 1 & $11(4 / 7)$ & 66 \\
Hispanic $(n=34)$ & 2 & 5 & 16 & $4.9 \pm 2.1$ & $4(1 / 3)$ & 25 & $4.4 \pm 2.4$ \\
Asian $(n=5)$ & 0 & 1 & 4 & 0 & $1(0 / 1)$ & 6 & $2.8 \pm 0.5$ \\
Unknown $(n=5)$ & 0 & 4 & 9 & 0 & $3(1 / 2)$ & 16 & $5.5 \pm 3.4$ \\
Total cohort $(n=1116)$ & 44 & 14 & 59 & 7 & $13(4 / 9)$ & 86 & $4.3 \pm 2.4$ \\
\hline
\end{tabular}

Race, based on birth certificate; $n$, the number of individuals in each race; private variants, those only seen in that race; promoter, promoter region; intron, intronic region; intron/exon junction, within 20 bp of intron/exon junction; exon (S/NS), exonic region (synonymous/nonsynonymous); SNPs/individual, average $\pm \mathrm{SD}$, significant differences in SNPs/individual include African-American $v s$ European-American $(p<0.001)$, African-American $v s$ Hispanic $(p<0.001)$, and African-American $v s$ Asian $(p<0.01)$; no differences in European-American $v s$ Hispanic $(p=0.2)$, European-American $v s$ Asian $(p=0.3)$, or Hispanic $v s$ Asian $(p=0.2)$ (intron/exon junction variants are also counted in the Intron variants column). The low number of variants in the Asian and unknown populations is attributable to the low number of Asian individuals and individuals of unknown ethnicity in this cohort. 
Table 2. Detection rate for SNPS with a given minimum allele frequency in SFTPB

\begin{tabular}{|c|c|c|c|c|c|c|c|}
\hline \multicolumn{8}{|c|}{ African-American $\mathrm{N}=197$} \\
\hline 2 & 0.086 & 0.123 & 0.159 & 0.257 & 0.307 & 0.328 & 0.411 \\
\hline 8 & 0.252 & 0.359 & 0.459 & 0.709 & 0.801 & 0.843 & 0.949 \\
\hline 16 & 0.343 & 0.484 & 0.611 & 0.884 & 0.949 & 0.972 & 0.999 \\
\hline 24 & 0.398 & 0.557 & 0.693 & 0.949 & 0.985 & 0.995 & $>.999$ \\
\hline 192 & 0.798 & 0.943 & 0.991 & $>.999$ & $>.999$ & $>.999$ & $>.999$ \\
\hline \multicolumn{8}{|c|}{ European-American $\mathrm{N}=875$} \\
\hline $\mathrm{n}$ & $0.1 \%(34)$ & $0.5 \%$ & $1 \%(12)$ & $5 \%(10)$ & $10 \%(7)$ & $20 \%(6)$ & $30 \%(5)$ \\
\hline 2 & 0.091 & 0.214 & 0.248 & 0.293 & 0.372 & 0.408 & 0.418 \\
\hline 48 & 0.389 & 0.798 & 0.887 & 0.982 & 0.998 & $>.999$ & $>.999$ \\
\hline 96 & 0.479 & 0.877 & 0.945 & 0.999 & $>.999$ & $>.999$ & $>.999$ \\
\hline 192 & 0.6 & 0.939 & 0.976 & $>.999$ & $>.999$ & $>.999$ & $>.999$ \\
\hline
\end{tabular}

The number within the parentheses indicates the number of variants with the specified minimum allele frequency; N, number of individuals; $n$, number of haploid genomes.

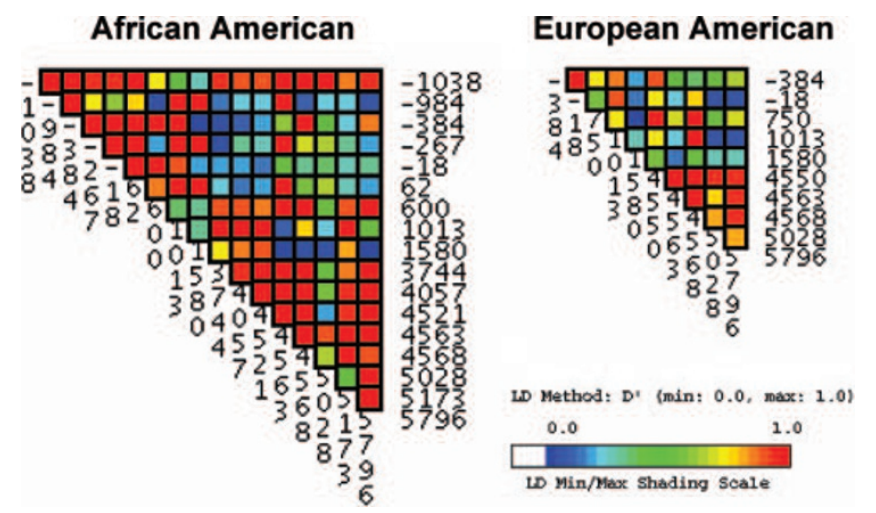

Figure 2. VG2 plot of $\mathrm{LD}\left(\mathrm{D}^{\prime}\right)$ within $S F T P B$ using common genotypes $(\mathrm{MAF}>5 \%)$ in African-American and European-American infants. Weak LD is present in both populations across the entire gene.

protein B function, we used two homology-based software tools, SIFT (Sorting Intolerant from Tolerant) (24) and PolyPhen (25). We found that eight of nine sites were not classified as intolerant or damaging. One site (genomic position 2558) in exon 5 that encodes either glycine or glutamic acid (G183E) was classified as probably damaging by Polyphen, but tolerated by SIFT, and is rare (MAF $0.1 \%$ ). The lack of definitively damaging or intolerant SNPs in this large cohort suggests strong, purifying, selective pressure against rare variants that encode dysfunctional surfactant protein $\mathrm{B}$, likely due to the critical role of the encoded protein in successful fetal-neonatal pulmonary transition (26). Despite evaluating a much larger cohort size (1116 versus 90 individuals from the Polymorphism Resource Discovery panel), these estimates are considerably lower than estimates of damaging exonic variants in 213 environmental genes (27).

To determine whether variants at intron-exon junctions might disrupt expression, we used a neural network applica- tion (http://www.fruitfly.org/seq_tools/splice-instrucs.html) trained to recognize potential human splice sites based on a large training set of known human splice sites. We found that the only common intron-exon junction SNP (genomic position 4550 , rs893159) was predicted to alter RNA splicing by creating a second acceptor site for exon 8 . The score for a second acceptor site increased from 0.47 to 0.78 when the minor allele was substituted, whereas the score for the predicted exon 8 acceptor site is 0.65 . This finding suggests that RNA splicing may be altered by this SNP.

To validate experimentally, a published mathematical simulation of the number of haploid genomes required to detect SNPs with minimum allele frequency greater than a given frequency (28), we performed 1000 race-stratified sampling iterations for SFTPB (Table 2). Our data for SFTPB confirm the theoretical prediction based on the standard neutral model of population genetics, show that a cohort size of $\leq 48$ haploid genomes will miss $11 \%-18 \%$ of SNPs with frequencies of $\geq 1 \%$ and provide direct evidence of the influence of population history on estimates of cohort size necessary to detect rare SNPs.

LD. Statistical power of genetic association studies may be increased, and genotyping costs decreased by identifying highly correlated tagSNPs. LD is a statistical measure of allelic correlation between polymorphisms. Using common genotypes (MAF $>5 \%$ ), we detected weak LD across $S F T P B$ despite its small genomic size (Fig. 2). In view of the effect of cohort size on LD, we randomly selected European-American cohorts similar in size to the African-American cohort and found similar results $(29,30)$. Using the tagger function in HAPLOVIEW, we were unable to capture rare variants when using common markers as tagSNPs. Using the Genome Variation Server maintained by Seattle SNPs (http:// 


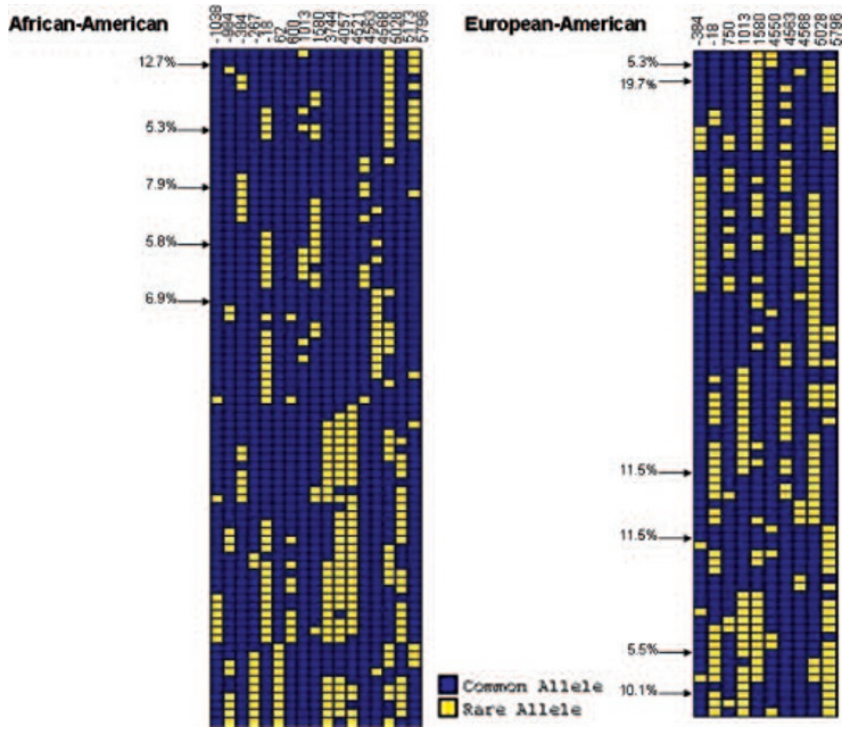

Figure 3. VH1 plot of common SFTPB haplotypes in African-American and European-American infants. Using 17 SNPs (African-American) and 10 SNPs (European-American), we estimated computationally 82 unique AfricanAmerican haplotypes and 80 unique European-American haplotypes. Most haplotypes (59/82 African-American haplotypes and 59/80 EuropeanAmerican haplotypes) were rare $(<1 \%)$. Arrows indicate haplotypes with frequencies $>5 \%$, and individual haplotype frequencies for common haplotypes are provided

gvs.gs.washington.edu/GVS), we found weak LD within $S F T P B$. Weak LD suggests that the genomic region that includes $S F T P B$ spans a recombination hot spot (14).

Haplotype diversity, estimation of background recombination rate, and recombination hot spot determination. We used PHASE with common genotypes (MAF $>5 \%$ ) to infer haplotypes (Fig. 3) and observed high haplotype diversity consistent with intragenic recombination. To determine whether SFTPB includes a recombination hot spot, we estimated recombination parameters into PHASE and calculated $\mathrm{BFs}$, a measurement of the strength of the evidence of a recombination hot spot (14). In the resequencing data alone, the intragenic recombination rate over background (Fig. $4 A$ ) and BF values (5.9 in European-American, 2.2 in AfricanAmerican) did not suggest a recombination hot spot. However, when we calculated the recombination rate and BFs for a 107-kb window flanking SFTPB in HapMap data, we found a 20- to 80-fold increase in the recombination rate within SFTPB (Fig. 4B), and BF values of 1353 in both populations. As suggested by comparison of BFs with background recombination rates in each of these cohorts (Fig. 5), the high intragenic recombination rate was not detected in the resequencing data because the recombination hot spot spans most of the resequenced region.

Molecular evolution. To test whether SFTPB variation is consistent with predictions from the neutral theory of molecular evolution, we used Tajima's D and the Fu and Li D* (Table 3). Both measures were consistently negative for both African-Americans and European-Americans, suggesting an excess of low frequency variation in $S F T P B$, although this trend was not significant. Using a sliding window approach (Fig. 6) (19), we found that the genomic region that encodes
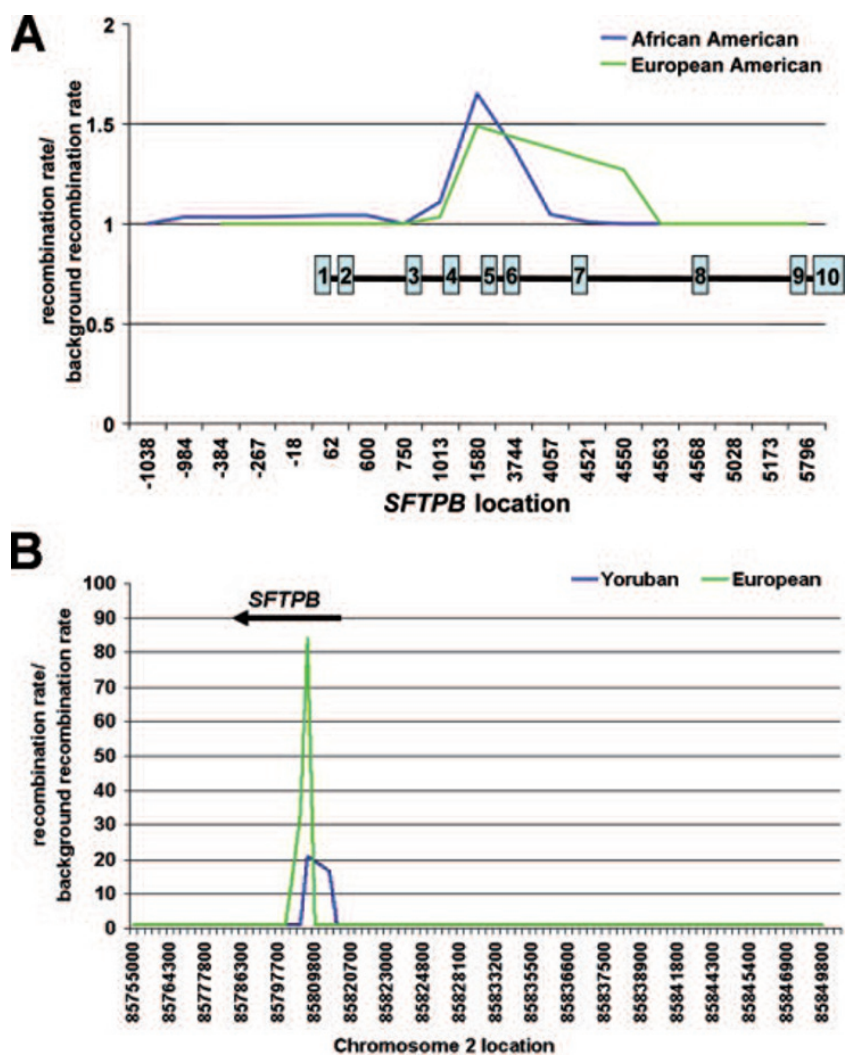

Figure 4. Changes in recombination rate $v s$ background recombination rate in the Missouri cohort within $S F T P B(A)$ and in the HapMap Project Yoruban and European cohorts (http://www.hapmap.org) near $( \pm 50 \mathrm{~kb}) \operatorname{SFTPB}(B)$. (A) In $S F T P B$, little change in recombination rate is detectable; positions of translated exons are shown in numbered blue boxes. (B) Near $S F T P B$, a 20to 80 -fold increase in recombination rate is present in $\triangle F T P B$.

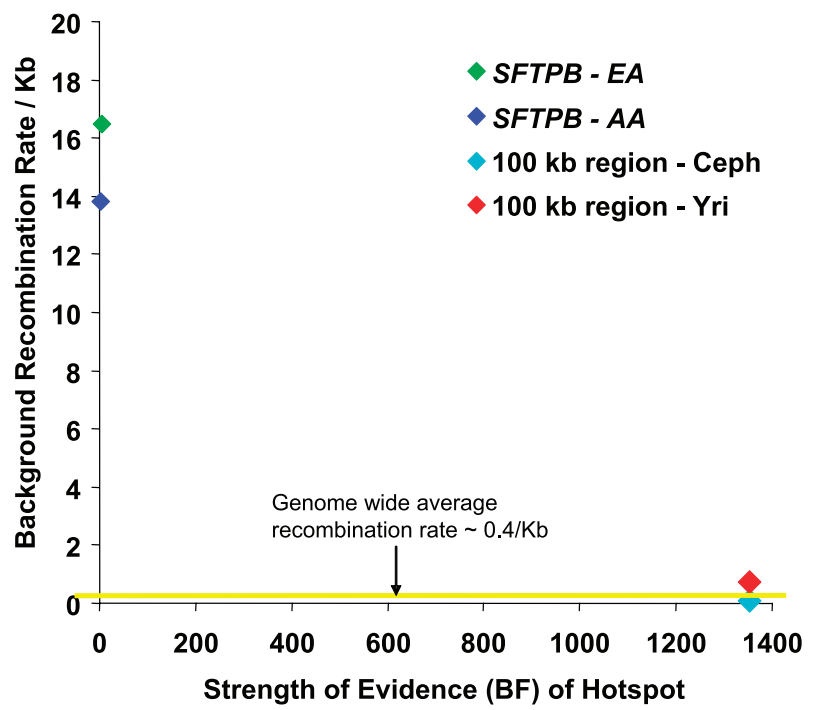

Figure 5. Strength of evidence of a recombination hot spot within $S F T P B$ using the Missouri cohort (SFTPB genomic region) and the HapMap cohort (http://www.hapmap.org/) (100-kb region that includes SFTPB). EA, European-American (Missouri cohort); AA, African-American (Missouri cohort); Yri, Yoruban population (HapMap Project); Ceph, European descent population (HapMap Project, data release \#21 as of July 2006).

mature surfactant protein B (exons 6 and 7) had the most negative values, consistent with negative selection against variation in these exons. To evaluate conservation across 
Table 3. Nucleotide diversity and neutrality tests in European-Americans and African-Americans for coding and noncoding regions and for synonymous and nonsynonymous SNPS

\begin{tabular}{|c|c|c|c|c|c|c|c|}
\hline Race & SNP & $\pi\left(\times 10^{-4}\right)$ & $\theta\left(\times 10^{-4}\right)$ & Tajima's D & $p$ & $\mathrm{Fu}$ and $\mathrm{Li} \mathrm{D*}$ & $p$ \\
\hline \multirow[t]{4}{*}{ EA } & $\mathrm{C}$ & 0.9 & 1.4 & -0.66 & 0.51 & -3.68 & 0.0002 \\
\hline & $\mathrm{NC}$ & 3.7 & 5.3 & -0.75 & 0.45 & -3.63 & 0.0003 \\
\hline & $\mathrm{S}$ & 0.01 & 0.2 & -0.79 & 0.43 & 0.40 & 0.689 \\
\hline & $\mathrm{T}$ & 4.1 & 6.6 & -1.0 & 0.32 & -5.99 & $<0.001$ \\
\hline \multirow[t]{3}{*}{ AA } & $\mathrm{C}$ & 1.7 & 2.7 & -0.83 & 0.41 & -1.87 & 0.062 \\
\hline & $\mathrm{NC}$ & 5.9 & 8.9 & -0.95 & 0.34 & -1.56 & 0.120 \\
\hline & $\mathrm{T}$ & 7.2 & 11.0 & -0.99 & 0.32 & -2.02 & 0.044 \\
\hline
\end{tabular}

C, coding; NC, noncoding; S, synonymous; NS, nonsynonymous; T, total; all $p$ values estimated assuming standard normal distribution; EA, EuropeanAmerican; AA, African-American. $\pi$ is a descriptive statistic for sequence diversity based on the number of chromosomes screened and the average allele frequency of the polymorphisms identified. $\theta$ is a descriptive statistic for sequence diversity based on the number of chromosomes screened and the number of polymorphisms observed. Tajima's D is a test statistic that compares the difference between $\theta$ and $\pi$ with theoretical expectations under an evolutionary model. The $\mathrm{Fu}$ and $\mathrm{Li} \mathrm{D}^{*}$ compares the difference between $\theta$ and the number of singleton SNPs observed with theoretical expectations under neutrality.

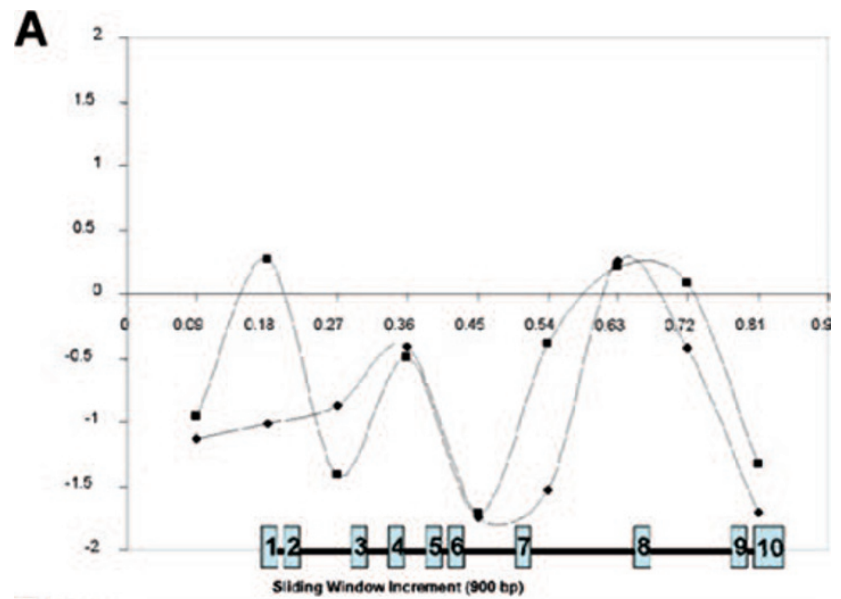

B

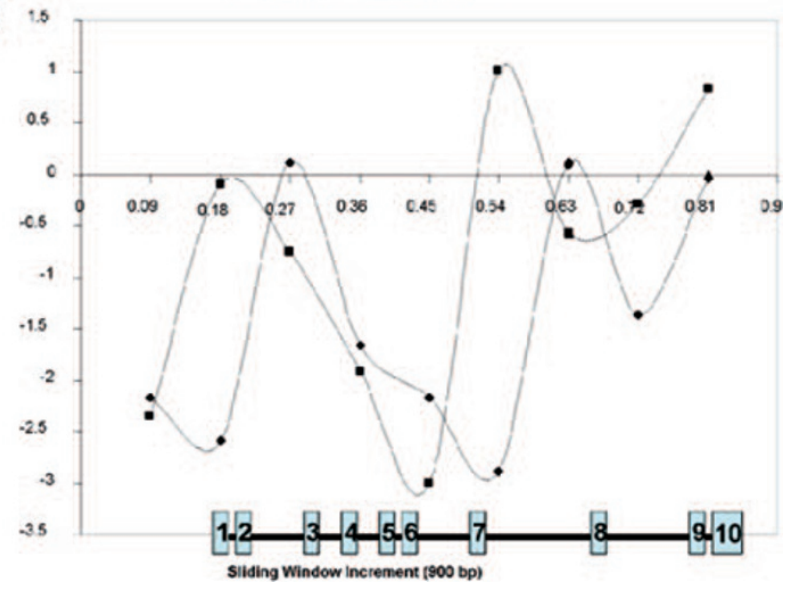

Figure 6. Comparison of Tajima's $\mathrm{D}(A)$ and the $\mathrm{Fu}$ and $\mathrm{Li} \mathrm{D}^{*}(B)$ across $S F T P B$ for the Missouri cohort using a 900-bp sliding window. EA $(\diamond)$, European-American infants; AA (ם), African-American infants; positions of translated exons shown in numbered blue boxes.

species, we compared dN/dS in this cohort with $S F T P B$ in Mus musculus (GenBank number NM147779). The overall $\mathrm{dN} / \mathrm{dS}$ ratio for this cohort was 2.0 (eight nonsynonymous and four synonymous sites). In a human-mouse comparison, SNAP determined the dN/dS ratio to be 0.94 (two nonsynonymous and 2.12 synonymous) across these two species, consistent with neutral evolution over time. The MacDonald-
Kreitman test was also consistent with neutral evolution $\left(\chi^{2}=\right.$ $0.43, p=0.51)$. These results suggest that although much of the variation in SFTPB is selectively neutral, the excess of low-frequency variation near the exons containing mature SFTPB may be attributable to the presence of a modest number of mildly deleterious polymorphisms subject to negative selective pressure.

\section{DISCUSSION}

Because neonatal respiratory distress syndrome is unambiguously associated with rare recessive $S F T P B$ mutations and is observed when $S F T P B$ expression is reduced by $>75 \%$ (2-5), $S F T P B$ is a candidate gene for neonatal respiratory distress syndrome. Previous studies using unrelated case-control designs or family-based association tests with genotypes at high-frequency polymorphic sites have suggested an association between genotypes or haplotypes and neonatal respiratory distress (31-33). To inform studies of genetic regulation of $S F T P B$, we adapted production level, polymerase chain reaction-based sequencing technology for comprehensive genetic variant discovery (7). We found high SNP density $(28,34)$, weak LD, and, using data from the HapMap Project, strong evidence of a recombination hot spot within SFTPB. The coincidence of high SNP density, excess low-frequency sites, and high recombination rate has been observed at other loci in Drosophila and humans (35-37), consistent with an increased mutation rate within recombination hot spots. These characteristics suggest that use of common $S F T P B$ haplotypes or tagSNPs will not capture statistically robust associations with disease-causing alleles in unrelated, genetically diverse casecontrol cohorts (38). Genetic bottlenecks in small populations will increase LD, but typically do so only for rare subsets of SNPs. LD between the higher frequency SNPs will not be substantially altered by bottlenecks in founder populations. Thus, at SFTPB, comprehensive resequencing in large casecontrol cohorts is advantageous for genetic association studies of neonatal respiratory distress syndrome because the elevated mutation rate enhances the frequency of rare deleterious mutations, whereas the high recombination rate makes LD between common SNPs too low for useful tagSNP selection. In 
view of the lack of common damaging exonic SNPs observed in $S F T P B$, association studies of neonatal respiratory distress syndrome will need to focus on regulatory variation. For example, our data using a neural network application trained to recognize potential human splice sites suggest that the intron-exon junction SNP at genomic position 4550 (rs893159) may alter RNA splicing, resulting in misprocessed or misdirected surfactant protein B and disrupting surfactant function. Our results also suggest the value of mechanistic studies in the genetic pathogenesis of SFTPB mutations. A second SNP in intron 2 (SNP 1013, rs3024798) may affect recombination rates within $S F T P B$ because it disrupts a motif in intron 2 (CCTCCCT $>$ CCTCCAT) that has been associated with recombination hot spot activity (39). Recombination rates correlate positively with mutation rates, so high recombination rate alleles may be more prone to the de novo SFTPB mutations seen in severe neonatal respiratory distress syndrome.

Acknowledgments. The authors thank members of the Seattle SNPs team (D.A. Nickerson, D.C. Crawford, M.J. Rieder, J. Sloan, and M. Eberle), R.H. Waterston, and H.R. Colten for helpful suggestions, and members of the Missouri DHSS (J. Eckstein, G. Land, M. Mosley, and J. Stockbauer) for collaboration.

\section{REFERENCES}

1. Whitsett JA, Weaver TE 2002 Hydrophobic surfactant proteins in lung function and disease. N Engl J Med 347:2141-2148

2. Nogee LM, Garnier G, Dietz HC, Singer L, Murphy AM, deMello DE, Colten HR 1994 A mutation in the surfactant protein B gene responsible for fatal neonatal respiratory disease in multiple kindreds. J Clin Invest 93:1860-1863

3. Cole FS, Hamvas A, Rubinstein P, King E, Trusgnich M, Nogee LM, deMello DE, Colten HR 2000 Population-based estimates of surfactant protein B deficiency. Pediatrics 105:538-541

4. Melton KR, Nesslein LL, Ikegami M, Tichelaar JW, Clark JC, Whitsett JA, Weaver TE 2003 SP-B deficiency causes respiratory failure in adult mice. Am J Physiol Lung Cell Mol Physiol 285:L543-L549

5. Merrill JD, Ballard RA, Cnaan A, Hibbs AM, Godinez RI, Godinez MH, Truog WE, Ballard PL 2004 Dysfunction of pulmonary surfactant in chronically ventilated premature infants. Pediatr Res 56:918-926

6. Hamvas A, Trusgnich MA, Brice H, Baumgartner J, Hong Y, Nogee LM, Cole FS 2001 Population-based screening for rare mutations: high-throughput DNA extraction and molecular amplification from Guthrie cards. Pediatr Res 50:666-668

7. Wilson RK, Ley TJ, Cole FS, Milbrandt JD, Clifton S, Fulton L, Fewell G, Minx P, Sun H, McLellan M, Pohl C, Mardis ER 2003 Mutational profiling in the human genome. Cold Spring Harb Symp Quant Biol 68:23-29

8. Hamvas A, Wegner DJ, Trusgnich MA, Madden K, Heins H, Liu Y, Rice T, An P, Watkins-Torry J, Cole FS 2005 Genetic variant characterization in intron 4 of the surfactant protein B gene. Hum Mutat 26:494-495

9. Holland PM, Abramson RD, Watson R, Gelfand DH 1991 Detection of specific polymerase chain reaction product by utilizing the $5^{\prime}-3^{\prime}$ exonuclease activity of Thermus aquaticus DNA polymerase. Proc Natl Acad Sci U S A 88:7276-7280
10. Devlin B, Risch N 1995 A comparison of linkage disequilibrium measures for fine-scale mapping. Genomics 29:311-322

11. Stephens M, Smith NJ, Donnelly P 2001 A new statistical method for haplotype reconstruction from population data. Am J Hum Genet 68:978-989

12. Stephens M, Donnelly P 2003 A comparison of bayesian methods for haplotype reconstruction from population genotype data. Am J Hum Genet 73:1162-1169

13. Barrett JC, Fry B, Maller J, Daly MJ 2005 Haploview: analysis and visualization of LD and haplotype maps. Bioinformatics 21:263-265

14. Crawford DC, Bhangale T, Li N, Hellenthal G, Rieder MJ, Nickerson DA, Stephens M 2004 Evidence for substantial fine-scale variation in recombination rates across the human genome. Nat Genet 36:700-706

15. Tajima F 1989 Statistical method for testing the neutral mutation hypothesis by DNA polymorphism. Genetics 123:585-595

16. Fu YX, Li WH 1993 Statistical tests of neutrality of mutations. Genetics 133:693 709

17. Nei M, Li WH 1979 Mathematical model for studying genetic variation in terms of restriction endonucleases. Proc Natl Acad Sci USA 76:5269-5273

18. Watterson GA 1975 On the number of segregating sites in genetical models without recombination. Theor Popul Biol 7:256-276

19. Carlson CS, Thomas DJ, Eberle MA, Swanson JE, Livingston RJ, Rieder MJ, Nickerson DA 2005 Genomic regions exhibiting positive selection identified from dense genotype data. Genome Res 15:1553-1565

20. Nei M, Gojobori T 1986 Simple methods for estimating the numbers of synonymous and nonsynonymous nucleotide substitutions. Mol Biol Evol 3:418-426

21. Ota T, Nei M 1994 Variance and covariances of the numbers of synonymous and nonsynonymous substitutions per site. Mol Biol Evol 11:613-619

22. McDonald JH, Kreitman M 1991 Adaptive protein evolution at the Adh locus in Drosophila. Nature 351:652-654

23. Tatusova TA, Madden TL 1999 BLAST 2 Sequences, a new tool for comparing protein and nucleotide sequences. FEMS Microbiol Lett 174:247-250

24. Ng PC, Henikoff S 2003 SIFT: Predicting amino acid changes that affect protein function. Nucleic Acids Res 31:3812-3814

25. Ramensky V, Bork P, Sunyaev S 2002 Human non-synonymous SNPs: server and survey. Nucleic Acids Res 30:3894-3900

26. Nogee LM 2004 Alterations in SP-B and SP-C expression in neonatal lung disease. Annu Rev Physiol 66:601-623

27. Livingston RJ, von Niederhausern A, Jegga AG, Crawford DC, Carlson CS, Rieder MJ, Gowrisankar S, Aronow BJ, Weiss RB, Nickerson DA 2004 Pattern of sequence variation across 213 environmental response genes. Genome Res 14:1821-1831

28. Kruglyak L, Nickerson DA 2001 Variation is the spice of life. Nat Genet 27:234-236

29. Weiss KM, Clark AG 2002 Linkage disequilibrium and the mapping of complex human traits. Trends Genet 18:19-24

30. Palmer LJ, Cardon LR 2005 Shaking the tree: mapping complex disease genes with linkage disequilibrium. Lancet 366:1223-1234

31. Floros J, Fan R, Diangelo S, Guo X, Wert J, Luo J 2001 Surfactant protein (SP) B associations and interactions with SP-A in white and black subjects with respiratory distress syndrome. Pediatr Int 43:567-576

32. Haataja R, Hallman M 2002 Surfactant proteins as genetic determinants of multifactorial pulmonary diseases. Ann Med 34:324-333

33. Floros J, Thomas NJ, Liu W, Papagaroufalis C, Xanthou M, Pereira S, Fan R, Guo X, Diangelo S, Pavlovic J 2006 Family-based association tests suggest linkage between surfactant protein B (SP-B) (and flanking region) and respiratory distress syndrome (RDS): SP-B haplotypes and alleles from SP-B-linked loci are risk factors for RDS. Pediatr Res 59:616-621

34. Zhao Z, Fu YX, Hewett-Emmett D, Boerwinkle E 2003 Investigating single nucleotide polymorphism (SNP) density in the human genome and its implications for molecular evolution. Gene 312:207-213

35. Begun DJ, Aquadro CF 1992 Levels of naturally occurring DNA polymorphism correlate with recombination rates in D. melanogaster. Nature 356:519-520

36. Aquadro CF, Bauer DuMont V, Reed FA 2001 Genome-wide variation in the human and fruitfly: a comparison. Curr Opin Genet Dev 11:627-634

37. Lercher MJ, Hurst LD 2002 Human SNP variability and mutation rate are higher in regions of high recombination. Trends Genet 18:337-340

38. Carlson CS, Eberle MA, Rieder MJ, Smith JD, Kruglyak L, Nickerson DA 2003 Additional SNPs and linkage-disequilibrium analyses are necessary for wholegenome association studies in humans. Nat Genet 33:518-521

39. Myers S, Bottolo L, Freeman C, McVean G, Donnelly P 2005 A fine-scale map of recombination rates and hotspots across the human genome. Science 310:321-324 